\title{
TIC, ASOCIATIVIDAD Y POTENCIAL TURISTICO, ELEMENTOS PARA PROMOVER EL DESARROLLO EN EL CATATUMBO
}

\section{ICT, ASSOCIATION AND TOURISM POTENTIAL, ELEMENTS TO PROMOTE DEVELOPMENT IN THE CATATUMBO}

\author{
MSc. Marilce Pacheco Carrascal*, MSc. Isbelia Karina Rincón Parada**, \\ Ph.D Sir Alexci Suarez Castrillon**
}

\author{
*Universidad Francisco de Paula Santander Ocaña, Facultad de Ciencias \\ Administrativas y Económicas, GIDSE. \\ Cra. 11 No. 40-75, Ocaña, Norte de Santander, Colombia. \\ 3167405295. mpachecoc@ufpso.edu.co
}

**Universidad Francisco de Paula Santander Ocaña, Facultad de Ingenierías, Grupo de Investigación Grucite. Vía Acolsure, Sede el Algodonal - Ocaña, Norte de Santander, Colombia. Tel.: (+57-7) 5690088, Ext.: 414. E-mail: ikrinconp

@ufpso.edu.co, sasuarezc@ufpso.edu.co

Resumen: En el presente artículo refleja el trabajo, producto de varios estudios que dos grupos de investigación de la Universidad Francisco de Paula Santander Ocaña, han llevado a cabo durante aproximadamente 7 años en torno al sector turismo en Ocaña y algunos municipios, con miras a conocer la situación de las empresas que directa o indirectamente prestan servicios conexos al turismo, así como la identificación y clasificación de los recursos y atractivos turísticos existentes, la capacidad operativa de los actores del turismo de Ocaña, Ábrego, La Playa y El Carmen, la creación de una página web y el análisis de la usabilidad de la misma; lo cual ha dado como resultado el planteamiento de estrategias organizacionales y comunicativas que permitan hacer de esta área geográfica un destino turístico con visibilidad y posibilidades de convertirla en una actividad económica que aporte al desarrollo regional, por medio de las TIC.

Palabras clave: Tecnologías de la información y la comunicación, asociatividad, potencial turístico, web 2.0.

\begin{abstract}
In the present article the work is reflected, product of several studies that two research groups of the Francisco de Paula Santander Ocaña University, have carried out during approximately 7 years around the tourism sector in Ocaña and some municipalities, with a view to knowing the situation of companies that directly or indirectly provide services related to tourism, as well as the identification and classification of existing resources and tourist attractions, the operational capacity of the tourism stakeholders of Ocaña, Ábrego, La Playa and El Carmen, the creation of a web page and the usability analysis of it; which has resulted in the approach of organizational and communication strategies that allow to make this geographical area a tourist destination with visibility and possibilities to turn it into an economic activity that contributes to regional development, through TIC.
\end{abstract}

Keywords: Technology of the information and communication, associativity, tourist potential, web 2.0. 


\section{INTRODUCCIÓN}

El turismo es un fenómeno social, cultural y económico relacionado con el movimiento de las personas a lugares que se encuentran fuera de su lugar de residencia habitual por motivos personales o de negocios/profesionales. Como tal, el turismo tiene efectos en la economía, en el entorno natural y en las zonas edificadas, en la población local de los lugares visitados y en los visitantes propiamente dichos. Debido a estos diversos impactos, la amplia gama y variedad de factores de producción requeridos para producir los bienes y servicios adquiridos por los visitantes y al amplio espectro de agentes involucrados o afectados por el turismo, es necesario adoptar un enfoque global del desarrollo, la gestión y la supervisión del turismo. Este enfoque es muy recomendable con miras a la formulación y puesta en práctica de políticas de turismo nacionales y locales, así como de acuerdos internacionales $\mathrm{u}$ otros procesos en materia de turismo.

La asociatividad económica y organizacional, mediante la estrategia del clúster, se convierte en una gran oportunidad para el incremento de la competitividad e innovación en el sector turístico.

Porter (1991) plantea que el fundamento de la estrategia competitiva es encontrar una posición dentro del sector donde actúa la empresa para defenderse de las fuerzas competitivas del mercado o para ejercer influencia para que actúen en su favor; es por ello que esta filosofía de trabajo busca incrementar la competitividad y la innovación del conjunto empresarial, que aunque realizan actividades diferentes, conforman el pul de servicios que el turista espera recibir cuando visita un lugar o un destino turístico.

Según informes de la Organización Mundial del Turismo, dentro de los sectores con mayor crecimiento y con tendencia a un crecimiento sostenible, es el de turismo; hecho que se convierte en una excelente oportunidad para el sector empresarial de las regiones con potencialidades para la ejecución de proyectos de asociatividad e integración, que permitan el desarrollo social y económico regional, bajo la concepción del clúster, como una alternativa organizacional y una filosofía de trabajo que promueve la innovación y la competitividad.
De otra parte, Jaime, Torres \& Álvarez (2014) indican que es de vital importancia considerar que los cambios tecnológicos han revolucionado al mundo y generado nuevas formas de organizar el trabajo y de ofrecer los productos-servicios, en pro de la satisfacción de los clientes y del mejoramiento de la competitividad; el sector turismo no ha sido ajeno a este fenómeno, por el contrario los estilos tradicionales de planear y organizar una salida vacacional ha tenido que avanzar incorporando los adelantos que la tecnología y la globalización ofrecen.

Parada, Castrillón \& Ortiz, (2017), indican que la web 2.0 engloba una serie de herramientas (blogs, redes sociales, galería de imágenes y videos, chats, entre otros) en las que se pone a prueba a todas las empresas y en especial aquellas que trabajan en el sector turismo, pues deben estar presentes si quieren sobrevivir y llegar a sus clientes potenciales; en este sentido se habla de la promoción turística en le web, según Middetlon (2001) esta promoción son todas las formas utilizadas para hacer que los clientes se den cuenta de los productos, que agudice sus deseos y estimule la demanda, así mismo Blanco (1990) añade que es una actividad integrada por un conjunto de acciones e instrumentos que cumplen la función de favorecer los estímulos al surgimiento y desarrollo del desplazamiento turístico, así como al crecimiento y mejora de las operaciones de la industria; todo lo anterior hace que el comercio del turismo, entre en una nueva era digital. (Pacheco, Arévalo, \& Rincón, 2015)

Dentro de los proyectos relevantes de turismo y Tic, se encuentra el trabajo realizado por un grupo de investigadores de la Universidad Simón Bolívar de Barranquilla-Colombia, cuya finalidad fue la construcción de un sistema de gestión y el desarrollo de un conjunto de estrategias para potenciar las ventajas del Caribe Colombiano como sector turístico e impulsar el desarrollo económico y social de esta región a fin de elevar el nivel de vida de sus habitantes mediante la utilización de las Tecnologías de la Información y las Comunicaciones; donde concluyen que el proyecto permitió aportar en el fortalecimiento de la asociatividad del gremio de actores del sector turístico del Caribe Colombiano; así mismo el caso de la ruta del vino del Maule en Chile, donde se realiza la difusión, reserva y venta de servicios ecoturísticos, apoyándose en las TIC y dejando 
claro la participación activa de la empresa, el estado y la universidad.

Bajo esta concepción y con el objeto de observar el potencial turístico de Ocaña, La Playa, Ábrego y El Carmen, el grupo de investigación GIDSE de la Universidad Francisco de Paula Santander Ocaña en convenio con la Cámara de Comercio de Ocaña, se dan a la tarea de investigar sobre este potencial y plantear la posibilidad de crear un clúster turístico, que permita ofrecer servicios de alta calidad, de manera sostenible, responsable, incluyente y participativa, generando un verdadero desarrollo económico; así mismo, se diseña la página web www.ocanaturistica.com, donde se da a conocer la riqueza cultural y turística de la región permitiendo el acceso desde un PC, Tablet o Smartphone, para la comunidad local e internacional.

Salguero (2006), considera que el desarrollo regional incluye toda acción público-privada, que en el marco de un territorio se lleva a cabo en búsqueda del bienestar colectivo y existen dos corrientes; las teorías de desarrollo endógeno consideran que los principales determinantes de desarrollo son los factores económicos y sociales de carácter interno de una región sin interdependencias externas de importancia y las teorías de desarrollo exógeno, donde predomina un enfoque más regional bajo diferentes cuestionamientos relacionados con la forma en que una región se interrelaciona con otras, ya sea a partir de su decidida contribución al desarrollo de otras como líder de estos procesos, o, en su defecto, desarrollándose al amparo de otras bajo determinados esquemas de dependencia.

Como lo plantea Hermansen (1969), el desarrollo económico de una región depende de tres procesos: Procesos de desarrollo cultural, de desarrollo social y político, administrativo e institucional, por lo tanto el desarrollo regional para la zona del Catatumbo debe orientarse bajo esta óptica.

Bonfante \& Castillo (2014), explican que los procesos de desarrollo cultural implican cambios de actitud y de criterios, fortalecimiento de creencias, valores y normas que regulan la conducta individual y social, y generación de elevadas aspiraciones de conciencia junto con nuevas creaciones en las artes y las letras; el desarrollo social incluye procesos sociales que abarcan diversos aspectos distributivos del desarrollo económico y de expansión de los sistemas de seguridad social, relacionados con la satisfacción de las necesidades humanas y mercantiles (nutrición, salud, vivienda, educación, recreación y servicios públicos); cambios de estructura de los grupos sociales y de los patrones de interacción o movilidad social y los procesos político-administrativos e institucionales están relacionados con los cambios en las posiciones de influencia y de poder y resultan del surgimiento de nuevos grupos sociales y de nuevas relaciones de intereses: formación de partidos políticos y organizaciones de todo tipo que defiendan los intereses colectivos.

\section{METODOLOGÍA}

Se utilizó la metodología cuantitativa de carácter descriptivo, la revisión documental, las visitas de observación, el registro fotográfico, las entrevistas y la aplicación de encuestas a los diferentes actores del turismo en Ocaña y su región, permitió el cumplimiento de cuatro etapas.

La primera etapa se enfocó a la identificación y clasificación de los recursos y atractivos turísticos en los cuatro municipios objeto de estudio como fue Ocaña, La Playa, Ábrego y El Carmen; la segunda, orientada a establecer la capacidad operativa de algunas empresas conexas al turismo, la tercera enfocada a evaluar el potencial turístico para la conformación del clúster, donde específicamente se seleccionan algunas de ellas y se hace el diseño de una página web que permita la interconectividad $\mathrm{y}$ sobre todo la visibilidad mundial de los recursos y atractivos turísticos de la región.

Para cumplir con la creación de la página Web donde se muestre la riqueza turística de la región, se realizó:

1. Una verificación de páginas en internet, para identificar las características que debe tener un sitio turístico; una vez seleccionadas las páginas, se realiza una tabla en donde se especifica los datos de usabilidad, accesibilidad, carga, redacción y contenido, utilidad y por último la presencia de la página en redes sociales.

Para esta revisión se utilizaron la aplicación Hera, WebSiteOptimization y WebPageTest.

2. Diseño de interfaz y la generación de una identidad gráfica de la página.

3. Diseño de navegación

4. Creación de la base de datos 
5. Prueba-corrección y publicación

\section{RESULTADOS}

\subsection{Identificación y clasificación de los recursos y atractivos turísticos.}

Ocaña y su región, está ubicada al nororiente de Colombia, en el departamento Norte de Santander, cuya temperatura oscila en promedio entre los $23^{\circ} \mathrm{C}$. Ocaña fue fundada en 1570 por Francisco Fernández de Contreras, en 1576 se trasladó a su actual ubicación y se le dio el nombre de Ocaña. Durante la época de la Independencia jugó papel de capital importancia, especialmente en la culminación de la Campaña del Bajo Magdalena y la Campaña Admirable, en 1813. El 9 de abril de 1828 se reunió en este lugar la Convención de Ocaña que discutiría la organización política y administrativa de la República; posteriormente, en 1857 , se erigió como municipio y hasta entonces ha sido un gran polo de desarrollo y centro comercial de esta región del Departamento Norte de Santander.

El municipio de Ábrego, se creó en 1530 con el nombre de El Llano de los Orejones; el 26 de Julio de 1580 con motivo de celebrarse la primera eucaristía se le denominó los Llanos de la Cruz. Estas fueron las primeras denominaciones que tuvo la población antes de la expedición de la Ordenanza $\mathrm{N}^{\circ} 32$ de 1930, que cambió definitivamente la última denominación a la de Ábrego, como homenaje a la heroína, doña Mercedes Ábrego.

La Playa de Belén, fue poblada en sus orígenes por pequeñas tribus indígenas conocidas con los nombres de Peritamas, Aspasicas, Curasicas y Aratoques. Según la versión de Monseñor García Benítez, los territorios del actual Municipio, en gran parte pertenecían a los dominios del cacique Patatoque. La fundación de la Playa de Belén, nace de la petición realizada por los señores Jesús Rueda, Juan Esteban Vega y Tiburcio Álvarez, al Excelentísimo Señor Obispo Fray Bernabé Rojas en el año de 1857 para la construcción de una capilla dedicada a San José, en el sitio llano alto. El 4 de diciembre de 1862 se bendijo la capilla y se le dio el nombre a la población como la Playa de Belén. (Pacheco \& Vergel, 2012); en 1996 obtuvo el premio al pueblo más bello de Colombia, destacando su arquitectura colonial y actualmente hace parte de la Red de Pueblos Patrimonio de Colombia y el municipio de El Carmen, el 16 de julio de 1686 fue creado con el nombre de estancia vieja de nuestra señora del Carmen, en 1700 tomó el nombre de estancia vieja y en 1843 la población toma el nombre de El Carmen. El municipio del Carmen es un presente vivo lleno de historia excepcional donde se han conjugado todo tipo de pasiones, de poder, gloria, amor y valentía.

Según la definición y clasificación del Ministerio de Comercio, Industria y Turismo (MCIT), los recursos turísticos son todos aquellos elementos que reúnen características que implican un atractivo para el turismo, bien sea de carácter natural de esparcimiento, de recreación, histórico o cultural, en la tabla 1 se presenta la relación de cada uno de los recursos que posee los municipios objeto de estudio, según la clasificación del MCIT.

\section{Tabla 1 identificación y clasificación de los recursos y atractivos turísticos.}

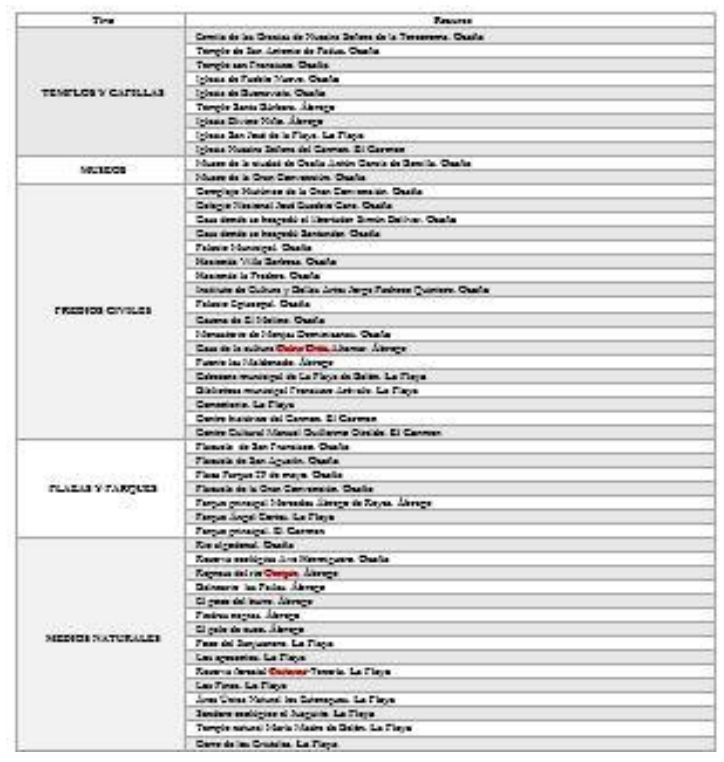

A pesar de que se encuentra con un gran potencial de recursos y atractivos turísticos, se deben hacer procesos de mejora en cuanto a la infraestructura y específicamente capacitar a los diferentes actores del turismo para la toma de conciencia de las ventajas de administrar con responsabilidad este potencial y el marketing para que la actividad turística sea un verdadero polo de desarrollo. Para el efecto de la capacitación se pueden implementar estrategias virtuales de tal manera que los empresarios del sector dispongan de su tiempo para realizar sus actividades cotidianas de manera más sencilla y práctica, como lo manifiesta Suárez Castrillón, González Castro \& Manzano Durán (2015) en su artículo análisis asistido por computador para la estrategia de aprendizaje e- 
portafolio, donde expresan que La importancia del uso de los cursos virtuales no sólo en los cursos o programas para establecer comunicación directa entre los interesados sino también en poder establecer procesos de investigación sobre las situaciones específicas para poder realizar planes de mejoramiento en torno a los resultados hallados.

\subsection{Capacidad operativa de los actores del turismo.}

Se indagó sobre la capacidad que tienen algunas empresas conexas al turismo como fue el caso de las agencias de Viajes y Turismo, consideradas como empresas comerciales, debidamente constituidas por personas naturales o jurídicas que se dediquen profesionalmente a vender planes turísticos y se encontró que en Ocaña existen cuatro que sólo ofrecen planes y paquetes turísticos a nivel nacional e internacional, como son Pitta Galván, Sanviatur, AerOcaña y Viajemos; del mismo modo se pudo indagar que en Ábrego, la Playa de Belén y el Carmen no existe ninguna agencia que desarrolle esta labor.

Con respecto a la capacidad hotelera, en Ocaña, se observó y verificó la existencia de 25 hoteles debidamente registrados ante la Cámara de Comercio y el Registro Nacional de Turismo, con 654 habitaciones dotadas con 514 camas sencillas, 459 dobles y 33 semidobles; en Ábrego se encontraron tres hoteles, dispuestos con 75 habitaciones con 34 camas sencillas, 61 dobles y 1 semidoble; en La Playa existen seis opciones de hospedaje con una capacidad de 40 habitaciones dotadas con 49 camas sencillas, 28 dobles y 12 semidobles y en El Carmen no existe ningún hotel registrado hasta el momento; allí este servicio es prestado por las familias Carmelitanas que tienen cuartos o casas disponibles que ofrecen este servicio a los visitantes.

Con relación a los restaurantes, como establecimientos públicos donde se ofrecen y sirven comidas y bebidas a las personas y hacen parte directa de la oferta de servicios turísticos, según datos suministrados por la Cámara de Comercio de Ocaña en cuanto al registro mercantil a finales del 2016, se pudo establecer y verificar la existencia de 46 restaurantes en la ciudad de Ocaña, en Ábrego se encontraron tres restaurantes, en La Playa de Belén tres restaurantes y en El Carmen tres restaurantes.

En cuanto a los sitios recreacionales, como lugares de descanso, recreación y esparcimiento juegan un papel fundamental en la presente investigación y se encontró que en Ocaña existen cuatro centros recreacionales, en Ábrego dos, siendo uno de propiedad de una comunidad religiosa, el cual facilitan para eventos más que todo espirituales; en el municipio de El Carmen hay uno y en La Playa existe el Parque Yaraguá que ofrece servicio de restaurante, hospedaje y cable vuelo, entre otros.

Los medios de transporte son fundamentales para la movilización de personas de un lugar de interés a otro con fines de trabajo u ocio. En Ocaña existen nueve agencias de transporte legalmente establecidas, responsables del trasporte de propios y visitantes. Es necesario aclarar que la empresa Cootransunidos despacha vehículos hacia el municipio de la Playa de Belén y Ábrego; Cootranshacaritama hacia los anteriores municipios además del municipio de El Carmen, esto en cuanto a los lugares de interés antes mencionados, además se desplazan para y desde Bucaramanga, Cúcuta, Aguachica, y empresas como Omega y Coopetrán lo hacen desde y para Bogotá, Medellín, Barranquilla y Cartagena.

Dentro de las opciones de transporte es necesario aclarar que hay tres que son empresas ocañeras, pero solamente dos de ellas cuentan con el registro nacional de turismo.

\subsection{Creación de la Página web.}

Las interfaces creadas, se fundamentan en la información recolectada en las etapas anteriores, como se muestra en la figura 1. Este sitio web www.ocanaturistica.com cuenta con un nombre de dominio propio que brinda fácil recordación y acceso y un traductor para que visitantes de habla inglesa puedan consultar y enterarse de las características y facilidades de los lugares incluidos dentro de cada uno de los municipios que hicieron parte del proyecto.

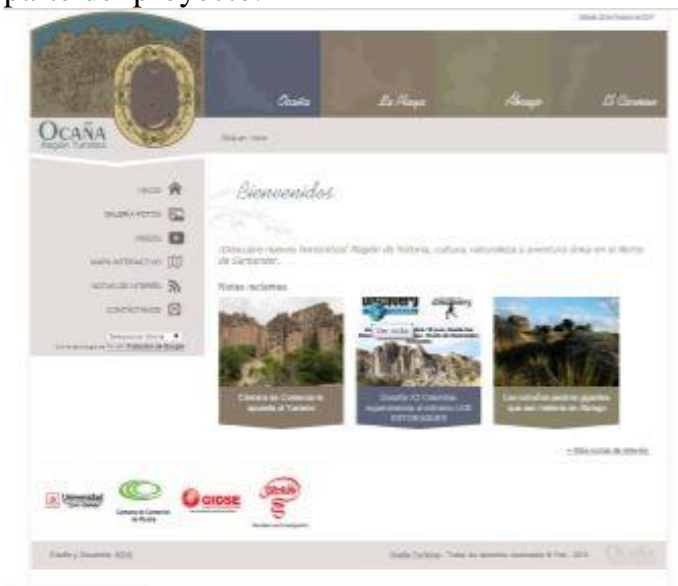

Figura 1: Interfaz página de turismo 
Posteriormente, se define la representación visual que incluye el logo (marca), diseño, tipografía y colores así mismo se decidió la inclusión de videos como herramientas para promover la imagen del destino turístico, con el objeto de enfatizar en los atributos, las características, las ideas y los valores que forman parte del destino. Para el diseño de navegación, se toma a Hassan y Martín (2002), quien considera que los elementos de navegación y orientación dentro de una página web tienen como función básica anunciar constantemente al usuario acerca de dónde se encuentra, qué relación tiene el nodo web que está visualizando respecto al resto de la arquitectura del website, dónde ha estado y hacia dónde puede ir, esto con el fin de no perder al usuario; por lo tanto, para el diseño de la página se realizó una navegación compuesta que brinda al usuario una buena experiencia a la hora de visitar el sitio (Pacheco, Arévalo, \& Rincón, 2015).

En la figura 2, se muestra el modelo entidad relación que se construyó tomando como base los distintos actores del turismo tales como: Agencias de Viajes y Turismo, Hoteles, Parqueaderos, Recursos turísticos, restaurantes, Sitios recreacionales y Empresas de transporte.

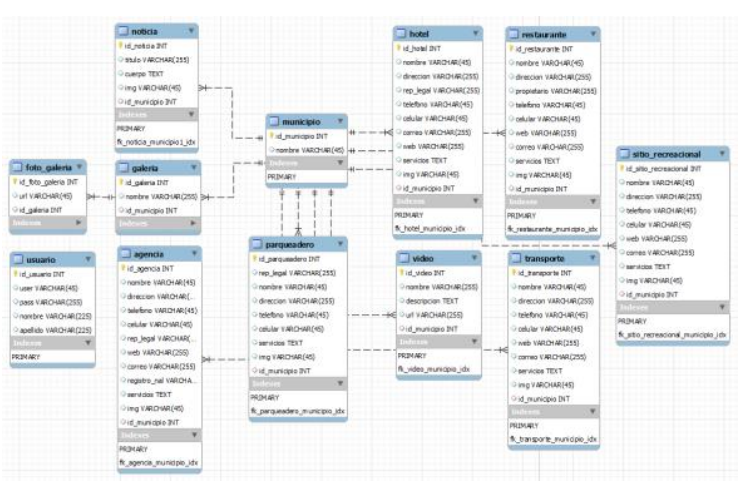

Figura 2: Modelo Entidad/Relación del Sitio

Posteriormente se programó el código que permite la consulta de la información almacenada en la base de datos, para lo cual se utilizaron técnicas y tecnologías actuales como HTML5, CSS3 y JavaScript para el Front-End (Lado del Cliente), para el Back-End (Lado del Servidor) se hizo uso de PHP y MySQL; se generó el panel de administrador y se publicó el sitio en un servidor remoto para realizar las pruebas de visualización, navegación y conectividad con la base de datos y hacer los ajustes necesarios y socializar públicamente la URL del sitio como es www.ocanaturistica.com.

\subsection{Consulta de usabilidad de la www.ocanaturistica.com.}

En noviembre de 2015 se realizó una consulta para evaluar la usabilidad del sitio y se encontró que durante el periodo de enero a octubre había ingresado 105.675 personas, a pesar de que aquí se incluye el periodo de prueba, ya que el lanzamiento por parte de la Cámara de Comercio de Ocaña, se hizo en mayo de 2015, lo que indica como se muestra en la figura 3 , que este sitio web potencializa el turismo y las visitas al destino; como lo plantean Middetlon (2001) y Blanco (1990), en donde la promoción turística por la web proporciona estímulos al surgimiento y desarrollo del desplazamiento turístico, así como al crecimiento y mejora de las operaciones de la industria; todo lo anterior hace que el comercio del turismo entre en una nueva era digital.

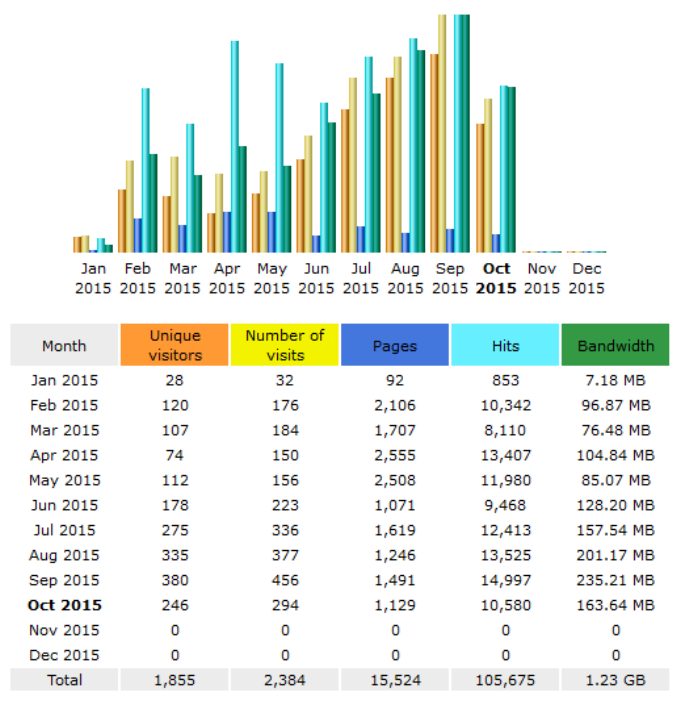

Figura 3: Estadísticas de visitas al 8 de octubre de 2015

\section{CONCLUSIONES}

Ocaña, junto los municipios de La Playa de Belén, El Carmen y Ábrego, cuentan con un gran potencial turístico que requieren la intervención y el trabajo mancomunado de los entes territoriales y estatales, la empresa privada, la comunidad y el sector educativo, para que se implementen planes y programas tendientes a la ejecución de estrategias que permitan el desarrollo regional bajo un 
enfoque sostenible e incluyente del sector turístico en esta zona geográfica del Catatumbo.

A pesar de su gran potencial en recursos y atractivos turísticos, se debe iniciar un proceso de mejoramiento e inclusión del turismo como actividad económica, para lo cual la organización empresarial mediante la filosofía de clúster es una alternativa que proporciona las herramientas necesarias para que los empresarios trabajen de manera asociativa en busca de la competitividad y el mejoramiento en la calidad de los servicios; así mismo se debe iniciar un proceso de concientización, cambio de cultura y capacitación de la comunidad en aspectos relacionados con el turismo y sus beneficios, para que el desarrollo de esta actividad fortalezca el tejido empresarial, mejore el nivel de vida de los habitantes, permita el ofrecimiento de servicios turísticos de calidad y convierta esta zona del país en un destino turístico a nivel mundial.

Otro aspecto de trascendencia es la urgencia de dar a conocer al mundo, el potencial turístico de esta zona, mediante el uso de las nuevas tecnologías de la información y la comunicación, siendo la web uno de los instrumentos claves para la comunicación de las marcas de destino y la comercialización de los servicios y productos inherentes al mismo, considerando que actualmente la mayor parte de la población tiene acceso a ellas. La web acerca al individuo a la información y facilita el acceso a los destinos turísticos.

\section{REFERENCIAS}

Blanco, M. (1990). Teoría general de turismo. Diana, México

Gasca. Maira Cecilia, Camargo. Luis Leonardo \& Delgado. Byron Medin. (2013). Modelo de negocios B2C a través de las tecnologías móviles en un centro comercial. Revista colombiana de tecnologías de avanzada ISSN: 1692-7257, 1(21).

Hassan Montero, Y., \& Martín Fernández, F. (1 de Diciembre de 2002). No solo usabilidad: revista sobre personas, diseño y tecnología. Recuperado el 29 de Octubre de 2015, de no solo usabilidad: revista sobre personas, diseño y tecnología:

http://www.nosolousabilidad.com/articulos/orie ntacion_usuario.htm

Hermansen, T. (1969). Development Poles and Development center in National and Regional
Development, United National Research Institute for Social Development. Ginebra.

Jaime Guzmán-Luna, Ingrid-Durley Torres, Juan Felipe Alvarez (2014). Propuesta de un generador de aplicaciones educativas basadas en televisión digital usando arquitectura de cómputo en la nube. Revista Colombiana de Tecnologías de Avanzada ISSN: 1692-7257 Volumen 2 - Número 24.

María Claudia Bonfante, Andrés Castillo (2014). Integración de sistema multi-agente, ontologías y procesos de negocios como marco tecnológico de la estrategia "gobierno en línea". Revista Colombiana de Tecnologías de Avanzada ISSN: 1692-7257 - Volumen 1 Número 23

Middleton, V. 2001 Marketing in travel and tourism. Butterworth-Heinemann, Oxford

Organización Mundial del Turismo OMT. (1994). http://media.unwto.org/es/content/entender-elturismo-glosario-basico. Recuperado el 22 de Enero de 2014, de http://media.unwto.org

Pacheco Carrascal, M., \& Vergel Quintero D. (2012). Capacidad turística de Ocaña y su región. Ocaña.

Pacheco Carrascal, M., Arévalo, B., \& Rincón Parada, I. K. (2015). La Web, estrategia para incentivar el turismo. Ingenio, 123-130.

Palmer, A. (2005). "The internet challenge for destination marketing organisations". En: Morgan, N., Pritchard, A. \& R. Pride: Destination Branding: Creating the Unique destination Preposition. Oxford: Elsevier.

Rincón, I. K. R., Suarez, A. S., \& Ortiz, E. A. S. (2017). Pensamiento crítico de los estudiantes que utilizan Facebook como nueva tecnología de aprendizaje. Revista colombiana de tecnologías de avanzada ISSN: 1692-7257 1(25).

Porter, M. E. (1991). Estrategia competitiva. Río de Janeiro Brasil: Campus.

Salguero Cubides, J. (2006). Enfoques sobre algunas teorías referentes al desarrollo regional. Bogotá: Sociedad deográfica de Colombia.

Suárez Castrillón, A., González Castro, Y. \& Manzano Durán, O. (2015). Análisis asistido por computador para la estrategia de aprendizaje e-portafolio. Revista Colombiana de Tecnológias de Avanzada, ISSN: 1692-7257 1(25), pp. 8-15. 